book is itself more than a field guide, giving fuller information about behaviour and reproduction than is necessary mərely for identification; the authors reasonably excuse its substantial bulk on the ground that travel in the desert anyhow necessitates a vehicle. The book gives a general account of all the bird species, resident and migratory, found in North Africa above about $21^{\circ} \mathrm{N}$. latitude, including the Canary Islands. The translation very elosely follows the original text, which the authors have not revised except to incorporate information from one recent source. They have, however, redrawn a dozen of the species distribution maps which are a feature of the work. The admirable illustrations by Paul Barruel, mostly in colour, are, of course, repeated.

Landsborough Thomson

\section{OUTSIDE THE NUCLEUS}

The Plastids

Their Chemistry, Structure, Growth and Inheritance. By John T. O. Kirk and Richard A. E. Tilney-Bassett. (A Series of Books in Biology.) Pp. xvi +608 . (London and San Francisco: W. H. Freeman and Company, 1967.) $110 s$.

In recent years the investigations of the fine structure and function of plastids by electron microscopists and molecular biologists have confirmed what has long been apparent to those pursuing the more conventional approaches of cytogenetics and physiology, namoly, that plastids have a dual role. They are both the seat of photosynthetic activity and the bearers of extrachromosomal hereditary information with its associated protein synthetic activity. The Plastids is a timely and comprehensive account of both of these activities in which the old and the new are fairly balanced, carefully assessed and put into historical perspective.

The book is divided into four parts: the first is a short general description of the nature of plastids; the second is concerned with the inheritance and gonetic autonomy of plastids illustrated by reference to the classical experiments on mutant and normal plastid differences in higher plants. An understanding of the nature of variegations and of the structure of chimeras is essential for a full appreciation of the significance of the breeding experiment and the observations on somatic segregation. The authors endeavour to provide this in the early chapters of the second part, although the combination of exhaustive detail, short sections and extensive cross-referencing makes heavy reading. Nevertheless, these are important chapters because the facts they contain provide the key to understanding how the separation of the chromosomal and extrachromosomal contributions to the determination of the plastid phenotype has been achieved. But while we are spared none of the details of the origin and structure of variegations and chimeras, the author's' explicit desire "not to burden the reader" leads to a vague account of the mathematical properties of the sorting-out from cells containing a mixture of two kinds of plastids, which is devoid of the simplest statistical expressions, formulae and concepts. The binomial and hypergeometric distributions which are the theoretical basis for all models of sorting-out are not oven mentioned.

The third part of the book gives the biochemical basis of plastid autonomy and plastid growth and accounts of the more recent evidence from electron microscopic and biochemical investigations. It contains a particularly good account of the genetical approach to the elucidation of the biosynthetic pathways which are involved in plastid development and in photosynthosis. Although every aspect of plastid structure and function in Chlamydomonas reinhardi is referred to somewhere in the book, the evidence for post-meiotic reassortment and recombination of extrachromosomal determixants of the plastid phenotype in this species has been omitted, even though it has an important bearing on some of the speculative discussion in the third part.

The final part of the book is a short summary of the contents of the first three parts followed by speculations about the possible future developments in the field of plastid research. Similar summaries at the end of sach chapter or part would have been invaluable in a book of this size and complexity. Unfortunately only three of the fifteen chapters and none of the parts have a concluding summary and many chapters end with a miscellany of material that could not find a place in their principal sub-sections. The book ends with three excellent indices for subjects, taxa and authors, respectively. These supplement the extensive references at the end of each chapter.

Taken as a whole, this book is an impressive work, beautifully illustrated and an invaluable source of references. Its publication is a landmark in the acceptance of the extrachromosomal contents of the cell as a partner of the chromosomal system in the heredity, growth and differentiation of green plants.

J. L. JINKS

\section{OBITUARIES}

\section{Professor H. J. Muller}

Hermann J. Muller, professor of zoology in the University of Indiana, Nobel laureate in 1946, died in Bloomington, Indiana, on April 5, 1967. He was born in Now York City in 1890, and graduated from Columbia College in 1910. Here he had been influenced by the cytologist Edmund B. Wilson and the embryologist Thomas Hunt Morgan, who was beginning his studies of the genetics of the vinegar fly, Drosophila melanogaster. Muller began the experimental analysis of heredity at Columbia with the first group of graduate students to devote themselves to work with Drosophila, and in the same year, 1915, he obtained his Ph.D. with a dissertation on "The Mechanism of Crossing-over", and became co-author of the book which initiated the new era in genetics, The Mechanism of Mendelian Heredity, by T. H. Morgan, A. H. Sturtevant, Muller and C. B. Bridges. While at Columbia he made two discoveries which determined the course of his future work: the recognition of what became known as cross-over suppressors, later shown to be caused by inversion of gene order in part of a chromosome, and this in turn led to the discovery of the first balanced lethal system which provided the clue to the explanation of the recurrent "mutations" which de Vries had discovered in the evening primrose Oenothera.

The insights thus provided into the nature of the genetic system led Muller to invent ingenious breeding systems for the quantitative study of the mutation process. Much of this work was carricd out at the University of Texas, where he was professor of zoology from 1920 to 1933. His paper on "The Problem of Genic Modification" provided the chief excitemont at the Fifth International Congress of Genetics held at Berlin in September 1927. It brought convincing exporimental proof that the mutation rate of genes of Drosophila could bo increased 150 -fold and more by treatment with X-rays. What set genetics on a new path was not merely the technical achiovement of inducing mutations, both those with lethal and with visible phenotypic effects, in measurable quantitative rates. Thore was, in particular, the application of these methods to the problems clearly onvisaged and pointed out by Muller of the analysis of the chemical and physical structure of the hereditary 